\section{P1-179 THE SOUTHAMPTON WOMEN'S SURVEY: FROM EPIDEMIOLOGY TO POLICY}

doi:10.1136/jech.2011.142976d.72

H Inskip,* K Godfrey, S Robinson, C Cooper, SWS Study Group. MRC Lifecourse Epidemiology Unit, Southampton, UK

Introduction Epidemiological studies inform understanding of influences on health. Findings can lead directly to policy changes, but such changes need assessing, using formal intervention studies.

Methods The Southampton Women's Survey is a longitudinal birth cohort with data collected on the mothers before conception. 12583 women aged 20-34 years were assessed when not pregnant; 3159 were then followed through pregnancy and the children are followed-up.

Results Maternal vitamin D levels in pregnancy were positively associated with markers of bone development in the children. Women's educational attainment was strongly related to the quality of their diets before conception, which in turn predicted the quality of the diets of their infants and children. Variations in infant diet were related to body composition at the age of 4 years. Our findings have led to intervention studies. Firstly, we are conducting a randomised controlled trial of vitamin D supplementation in pregnancy (MAVIDOS). Secondly, in relation to our diet quality findings, we are conducting a complex intervention, in collaboration with local policy makers, in which staff working in centres for women and children in disadvantaged areas are trained to engage in "healthy conversations' with young women visiting the centres, to enable them to improve their diets and lifestyles (Southampton Initiative for Health). A school intervention (LifeLab) is also being developed. Conclusion The Southampton Women's Survey, a large epidemiological study, has led to the development of interventions to improve health of women and their children. These are being evaluated to inform policy, locally, nationally, and internationally.

\section{P1-180 COULD INFLUENZA TRANSMISSION BE REDUCED BY RESTRICTING MASS GATHERINGS? AN EVIDENCE REVIEW}

doi:10.1136/jech.2011.142976d.73

\footnotetext{
${ }^{1,3} \mathrm{D}$ Ishola, ${ }^{* 1,2} \mathrm{~N}$ Phin. ${ }^{1}$ Health Protection Agency, London, UK; ${ }^{2}$ University of Chester,
} Chester, UK; ${ }^{3}$ University College London, London, UK

Introduction Droplet spread and direct contact are crucial factors in influenza transmission. Mass gatherings (MG) seem to provide ideal conditions for transmission. This systematic review examined the evidence for an association between MG and influenza transmission, and assessed whether restricting $M G$ is effective at reducing or preventing transmission.

Methods The published literature to July 2010 was reviewed by searching major databases (Pubmed, EMBASE, Scopus, and CINAHL), producing a combined list of 1670 articles that was sifted for relevance by title, abstract, and full-text assessment. A narrative approach was adopted for data synthesis.

Results 18 papers met the inclusion criteria, covering MG of varying sizes and settings, and including 5 observational studies, nine outbreak reports, three event surveillance reports, and a quasiexperimental study. There is evidence that some MG are associated with increased risk of influenza transmission. MG can also be "seeding" events for introducing new strains to an area, and may instigate community transmission in the early stages of a pandemic. Restriction of MG, in combination with other social distancing interventions, may help reduce transmission, but evidence is lacking on the effect of MG restriction alone.

Conclusion The evidence suggests that crowd density and event duration may be the key characteristics of an MG that determine the risk of influenza transmission. The type of venue (indoor or outdoor) also seems crucial. These considerations could potentially underlie the development of evidence-based, risk-dependent, policy-making frameworks for restricting $M G$ in pandemic situations. Voluntary rather than legislated restrictions may be suitable.

\section{P1-181 MORTALITY AND EPIDEMIOLOGICAL TRANSITION IN BANGLADESH: LESSONS AND EXPERIENCES TO THE DEVELOPING COUNTRIES IN THE NEW MILLENNIUM}

doi:10.1136/jech.2011.142976d.74

M Islam.* University of Dhaka, Dhaka, Bangladesh

Background This study aims to examine the stages of mortality and epidemiologic transition in Bangladesh where detailed studies are not well documented but significant decline in fertility and mortality have been achieved at a rather low level of income.

Methods With the application of time-series and cross sectional analyses by using the nationally representative data (Bangladesh Demographic and Health Survey, 1993-2007, Sample Vital Registration System, 1981-2007) this study investigates the patterns, levels and trends of mortality and morbidity and the causes of death Author's own field work-a mix of quantitative and qualitative methods have been referred for disease or health transition at grassroots level.

Results Study shows that the changing pattern of diseases observed over recent years, from acute infectious and deficiency diseases to the chronic non-communicable diseases, is a continuous process of transformation with some diseases disappearing and others appearing or reappearing. Although infectious diseases are still an important public health concern but non-communicable diseases are also coming to the forefront as causes of illness and death, where it is possible to control many communicable diseases.

Conclusion Bangladesh is now entering well into the third phase of mortality transition while the epidemiological transition where the place is taking in between the age of degenerative and manmade diseases followed by the transitional variant of delayed model. This study may help to understand the process of the stated transitions in Bangladesh comparing with other developing countries identifying the changes and challenges to achieve the MDGs and further for appropriate policy options.

\section{P1-182 A TAXONOMY OF BIAS IN SYSTEMATIC REVIEWS}

doi:10.1136/jech.2011.142976d.75

K Jacobsen. ${ }^{*}$ George Mason University, Fairfax, Virginia, USA

Introduction The goal of a systematic review is to compile and summarise all relevant reports on one well-defined topic. Selection bias is minimised by carefully defining search strategies, keywords, and eligibility criteria. However, remaining sources of bias may lead to the omission or under representation of relevant literature.

Methods The objective of this study was to identify and classify sources of selection bias in systematic reviews. The search methods and stated limitations of more than 50 systematic reviews were examined and catalogued.

Results Some forms of bias result from systemic barriers in the research dissemination process. These include (1) pipeline biases, such as those that hinder the dissemination of null association reports, and (2) indexing biases, such as the exclusion of non-English journals from major abstract databases. Other forms of bias result from methodological choices made by researchers. These include (3) search biases, such as those related to the use of hand-searches and 